\title{
The fecal microbiota of semi-free-ranging wood bison (Bison bison athabascae)
}

\author{
J Scott Weese ${ }^{1 *}$, Todd Shury ${ }^{2,3}$ and Murray D Jelinski ${ }^{4}$
}

\begin{abstract}
Background: The intestinal tract harbours a complex and diverse microbial population that is important for health, yet has been poorly described in many species. This study explored the fecal microbiota of semi-free-ranging Wood bison (Bison bison athabascae).

Results: A total of 2081936165 rRNA (V4) sequences from 40 bison were evaluated. CatchAll analysis of richness predicted a mean of 10685 species per sample (range 5428-24764, SD 4136). Diversity was high, with an average inverse Simpson's index of 31.78 (SD 15.3, range 8.55-86.7). Twenty-one different phyla were identified; however, only Firmicutes and Proteobacteria, Actinobacteria accounted for $>1 \%$ of sequences. Two distinct population clusters (Group $A, n=19$ and Group $B, n=21$ ) were evident based on both community membership and population structure. Group $A$ had a significantly lower relative abundance of Actinobacteria (6.4 vs $11.8 \%, P=0.002$ ), Chloroflexi ( 0.002 vs $0.013 \%, P=0.014)$, Gemmatimonadetes $(0.007$ vs $0.15 \%, P=0.038)$ and Proteobacteria (18.7 vs $42.5 \%$, $P=<0.0001)$ and a greater relative abundance of Firmicutes ( 70.9 vs $39.3 \%, P<0.0001)$ than Group B. Within Group B, Alphaproteobacteria was the most common class of Proteobacteria (28\% of all sequences), while Caulobacteraceae (18.5\%), Pseudomonadaceae (3.5\%), Hyphomicrobiaceae (3.5\%), Alcaligenaceae (3.1\%) and Xanthomonadaceae (2.6\%) were the most abundant families.

The twenty (3.1\%) most abundant genera accounted for $71 \%$ of sequences. No operational taxon units (OTUs) were found in all samples at a relative abundance of $1 \%$ or greater. One OTU (Clostridium cluster XI) was present at 1\% or more in all Group A samples, with two other Clostridium cluster XI OTUs in 18/19 (95\%) samples. No OTUs were found at that abundance in all Group B sample, but an unclassified Lachnospiraceae was present in 20/21 (95\%) and Clostridium cluster XI and Brevundimonas were found in 19 (90\%) samples.
\end{abstract}

Conclusions: The fecal microbiota of Wood bison is rich and diverse. The presence of two distinct populations not associated with housing, age or gender suggest that enterotypes, distinctly different microbial population compositions that can achieve the same ultimate function, might be present in bison, as has been suggested in humans.

Keywords: Bison, Microbiota, Microbiome, Intestinal

\section{Background}

The American bison (Bison bison) is a ruminant herbivore that is found in the wild in regions of western Canada and the United States and also raised in captivity as a food animal. Wood bison (Bison bison athabascae) are a geographic variant of Plains Bison (Bison bison bison) that are morphologically larger, but not genetically distinct and have historically inhabited areas in northern Canada

\footnotetext{
* Correspondence: jsweese@uoguelph.ca

${ }^{1}$ Department of Pathobiology and Centre for Public Health and Zoonoses, Ontario Veterinary College, University of Guelph, Guelph, ON, Canada Full list of author information is available at the end of the article
}

and Alaska. While they are raised commercially, little is known about the biology and health of bison compared to other domestic food animals. Some information can be inferred from other ruminants, but there are potentially important physiological and microbiological differences $[1,2]$ that indicate the need for specific study.

The gastrointestinal microbial population, the microbiota, is receiving increasing study in many species because of its role in health and various diseases, as well as its complex interaction with the body. Despite the known importance of the intestinal microbiota in many other species, there has been limited study in bison. Culture-based 
methods have evaluated the fecal and ruminal microbiota of bison [1-4], but it is now clear that such approaches have significant inherent limitations for characterization of the microbiota. Culture-based methods provide a very superficial understanding of the intestinal microbiota because of its complexity, richness (number of different organisms), the fastidious nature of many microorganisms, the potential for profound culture bias (over-representation of bacteria that grow well under typical culture conditions) and because a large percentage of the microbiota is currently unculturable. While not without their own potential biases, culture-independent methods, particularly next generation sequencing, have now become the standard for assessment of complex polymicrobial environments.

Understanding the microbiota of normal animals can be important for assessing the role of the microbiota in disease, as well as the impact of various management practices (e.g. diet change, antimicrobial administration) on the microbiota. Accordingly, the objective of this study was to evaluate the fecal microbiota of members of a semi-free-ranging extensively managed wood bison herd using a culture-independent next generation sequencebased approach.

\section{Methods}

\section{Study population and sample collection}

Fecal samples were collected from 40 semi free-ranging wood Bison, located in a 60 square kilometre fenced area within Elk Island National Park near Edmonton, Alberta, Canada. Bison are fenced behind a 2 metre high fence, but are essentially managed as wildlife within a national park. Animals were a mix of ages and sexes, but were fed the same hay diet for approximately one month prior to sampling. Bison originated from three separate pens in which animals were segregated by age and sex. Pen A housed 7 male calves (approx. 7 months old), pen $\mathrm{B}$ housed 20 adult females ( $>3$ years of age) and pen $C$ housed 13 adult males ( $>3$ years of age). None of the animals had any history of antimicrobial exposure within the past two years. Fecal samples were collected per rectum during routine handling in a custom designed facility with alleyways and squeeze chute, which occurs biennially to allow management of surplus animals. Fecal samples were frozen at $-20^{\circ} \mathrm{C}$ prior to analysis. This study was approved by the University of Saskatchewan Research Ethics Office (protocols 20130032 and 20130037).

\section{DNA extraction and quality control}

DNA was extracted from fecal samples using a commercial kit according to the manufacturer's instructions $^{\mathrm{a}}$. DNA quantity and quality were accessed by spectrophotometry ${ }^{\mathrm{b}}$.

\section{$16 \mathrm{~S}$ rRNA gene amplification and sequencing}

The V4 region of the 16S rRNA gene was amplified using the primers forward S-D-Bact-0564-a-S-15 (5'AYTGGGYDTAAAGNG-3') and reverse S-D-Bact0785-b-A-18 (5'-TACNVGGGTATCTAATCC-3') [5] as has been previously described [6]. PCR products were evaluated by electrophoresis in $2 \%$ agarose gel and purified with the Agencourt AMPure XP system. ${ }^{\mathrm{c}}$ Sequencing of the library pool was performed at the University of Guelph's Advanced Analysis Centre using an Illumina MiSeq $^{\mathrm{d}}$ and $2 \times 250$ chemistry.

\section{Bioinformatics}

The mothur package of algorithms (v1.32.1) was used for analysis [7]. Paired end reads were aligned. Sequences were aligned with the Silva 16S rRNA reference database (www.arb-silva.de) [8]. Sequences that were $>244$ bp or $<239$ bp in length, contained any ambiguous base calls or long runs ( $>8 \mathrm{bp}$ ) of holopolymers or did not align with the correct region were removed. Chimeras were identified using uchime [9] and eliminated. CatchAll was used to assess species richness [10]. Taxonomy was assigned using the RDP taxonomy database (http://rdp.cme.msu.edu/index.jsp). Sequences were binned into operational taxon units (OTUs) at a 3\% dissimilarity level.

Subsampling was performed to normalize sequence numbers for further comparison. This consisted of random selection of a number of sequences from each sample that corresponded to the lowest sequence abundance of all samples. Coverage was assessed using Good's coverage and visualized using rarefaction curves. Population diversity was described using the inverse Simpson's index and evenness was assessed using Shannon's evenness. Community membership was compared using the traditional Jaccard index, while community structure was assessed using the Yue \& Clayton measure of dissimilarity. The core microbiota was assessed through identification of OTUs present in all samples at a minimum relative abundance of $1 \%$.

Visual assessment of dendrograms identified two distinct groups, which were studied further. Relative abundances and alpha diversity indices were compared using t-test. The parsimony test was applied to the Jaccard and Yue \& Clayton trees. Dissimilarity was also visualized using principal coordinate analysis $(\mathrm{PCoA})$, and the statistical significance of the observed spatial separation was assessed using Analysis of Molecular Variance (AMOVA) and analysis of similarity (ANOSIM). A P value of $<0.05$ was considered significant for all comparisons.

\section{Results}

A total of 2081936 V4 16S RNA gene sequences from 40 bison passed all quality control filters. The number of 
sequences per sample ranged from 17667 to 106284 (mean 52049, SD 16896). CatchAll analysis of richness predicted a mean of 10685 species per sample (range 5428-24764, SD 4136). Good's coverage ranged from 0.943-0.976 (mean 0.962, SD 0.0097). Rarefaction curves based on subsampling of 17667 sequences per sample are displayed in Additional file 1: Figure S1. Diversity was high, with an average inverse Simpson's index of 31.78 (SD 15.3, range 8.55-86.7). Shannon's evenness values were an average of 0.60 (SD 0.046, range 0.477-0.683).

Two distinct clusters (Group A, n = 19 and Group B, $\mathrm{n}=21$ ) were evident visually with both the Yue \& Clayton (Figure 1) and Jaccard (data not shown) dendrograms. This was also evident visually using PCoA (Figure 2). Community membership and structure were significantly different based on parsimony test applied to both the Yue \& Clayton $(P=0.034)$ and Jaccard $(P=<0.001)$ trees. Significant differences in community structure were also observed with AMOVA $(P<0.001)$ and analysis of similarity (ANOSIM) $(P<0.001)$. There was no difference in evenness between groups (0.644 vs 0.640 , $P=0.78$ ) and while there was not a significant difference in diversity (inverse Simpson's, 36.7 vs 27.3) it approached significance $(P=0.06)$. There was no association between pen-of-origin and Group $(P=0.53$, Table 1$)$.
Twenty-one different phyla were identified (Table 1, Figure 3); however, only three (Firmicutes, Proteobacteria, Actinobacteria) accounted for $>1 \%$ of sequences overall. There were numerous significant differences in relative abundances of different phyla between groups (Table 2), with the most pronounced differences being with Firmicutes and Proteobacteria. Group A had a significantly lower relative abundance of Actinobacteria (6.4 vs $11.8 \%, P=0.002)$, Chloroflexi ( 0.002 vs $0.013 \%$, $P=0.014)$, Gemmatimonadetes $(0.007$ vs $0.15 \%, P=$ 0.038 ) and Proteobacteria ( 18.7 vs $42.5 \%, P=<0.0001$ ) and a greater relative abundance of Firmicutes (70.9 vs $39.3 \%, P=<0.0001)$ than Group B. Six hundred fifty two genera were identified, with Clostridium cluster XI the most abundant overall (13\%), followed by Brevundimonas (9.4\%). The predominant genera in each group are presented in Table 3.

Within Group B, Alphaproteobacteria was the most common class of Proteobacteria (28\% of all sequences) followed by Gammaproteobacteria (8.0\%) and Betaproteobacteria (6.0\%). Caulobacterales (18.5\%), Rhizobales (7.4\%), Burkholderiales (5.9\%), Pseudomonadales (3.9\%) and Xanthomonadales (1.6\%) were the main orders, while Caulobacteraceae (18.5\%), Pseudomonadaceae (3.5\%), Hyphomicrobiaceae (3.5\%), Alcaligenaceae (3.1\%) and

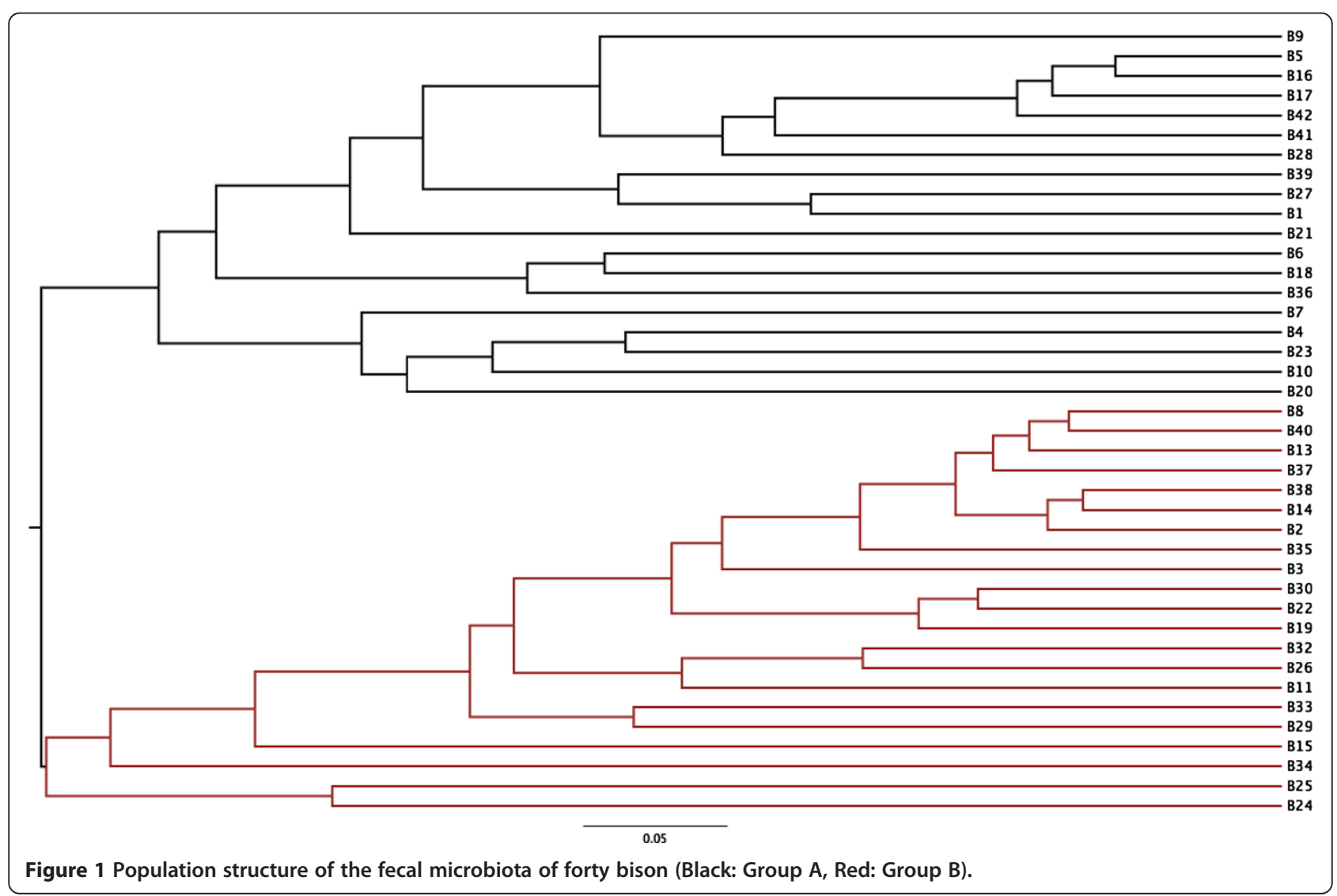




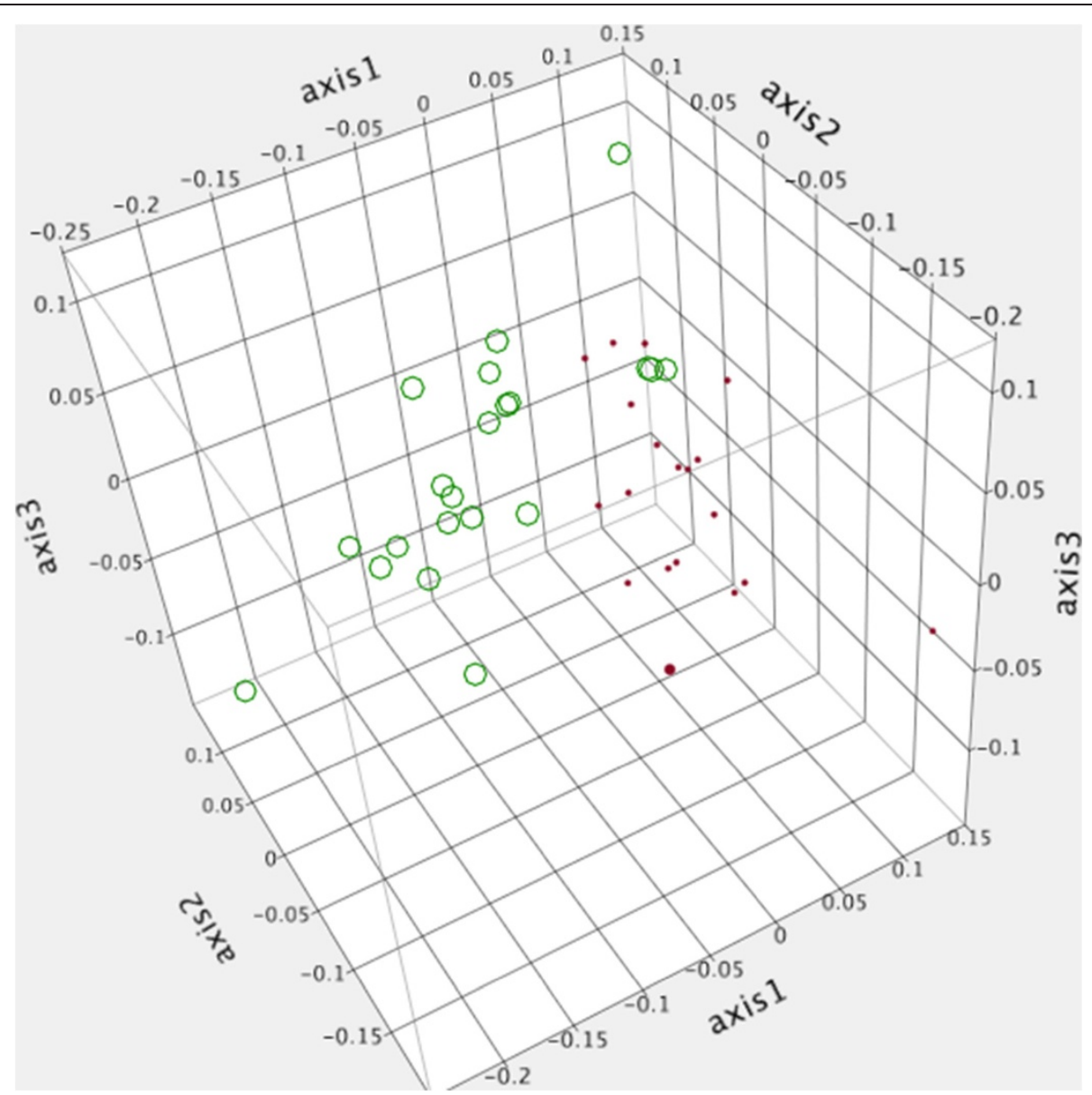

Figure 2 Principle coordinate analysis of the bacterial microbial population of forty bison, separated into two different enterotypes (Group A: red, Group B: green).

Xanthomonadaceae (2.6\%) were the most abundant families.

A total of 652 different genera were identified. Most were rare, with the twenty (3.1\%) most abundant genera accounting for $71 \%$ of total sequences. No OTUs were found in all samples at a relative abundance of $1 \%$ or greater. One (Clostridium cluster XI) was found at a relative abundance of at least $1 \%$ in $38 / 40$ (95\%) samples, two (Clostridium cluster XI and an unclassified Lachnospiraceae) in 37/40 (93\%) samples and 4 (two Clostridium cluster XI, unclassified Lachnospiraceae, Brevundimonas) in $33 / 40(83 \%)$ samples.

Table 1 Pen classification of two different groups of fecal microbiota in bison

\begin{tabular}{lllll}
\hline Pen & Description & Group A & Group B & Total \\
\hline A & Male calves & 2 & 5 & 7 \\
B & Adult females & 10 & 10 & 20 \\
C & Adult males & 7 & 6 & 13 \\
Total & & 19 & 21 & 40 \\
\hline
\end{tabular}

When Group A was analysed separately, one OTU (Clostridium cluster XI) was present at $1 \%$ or more in all samples, with two other Clostridium cluster XI OTUs were present in 18/19 (95\%) samples. No OTUs were found at that abundance in all Group B sample, but an unclassified Lachnospiraceae was present in 20/21 (95\%), while a Clostridium cluster XI and Brevundimonas were found in 19 (90\%) samples.

\section{Discussion}

The fecal microbiota of bison contains a rich and diverse microbial population. It was rather surprising to find two distinct subpopulations since animals harbouring these subpopulations had been co-housed and fed the same haybased diet for approximately one month. Typical factors associated with gut microbiota alteration such as differences in diet, management, diet change, age, antimicrobial administration and gastrointestinal disease were not present, there was no association with age or gender, and no other differences between these groups were evident. Reasons for this are unclear but one potential relates to the 


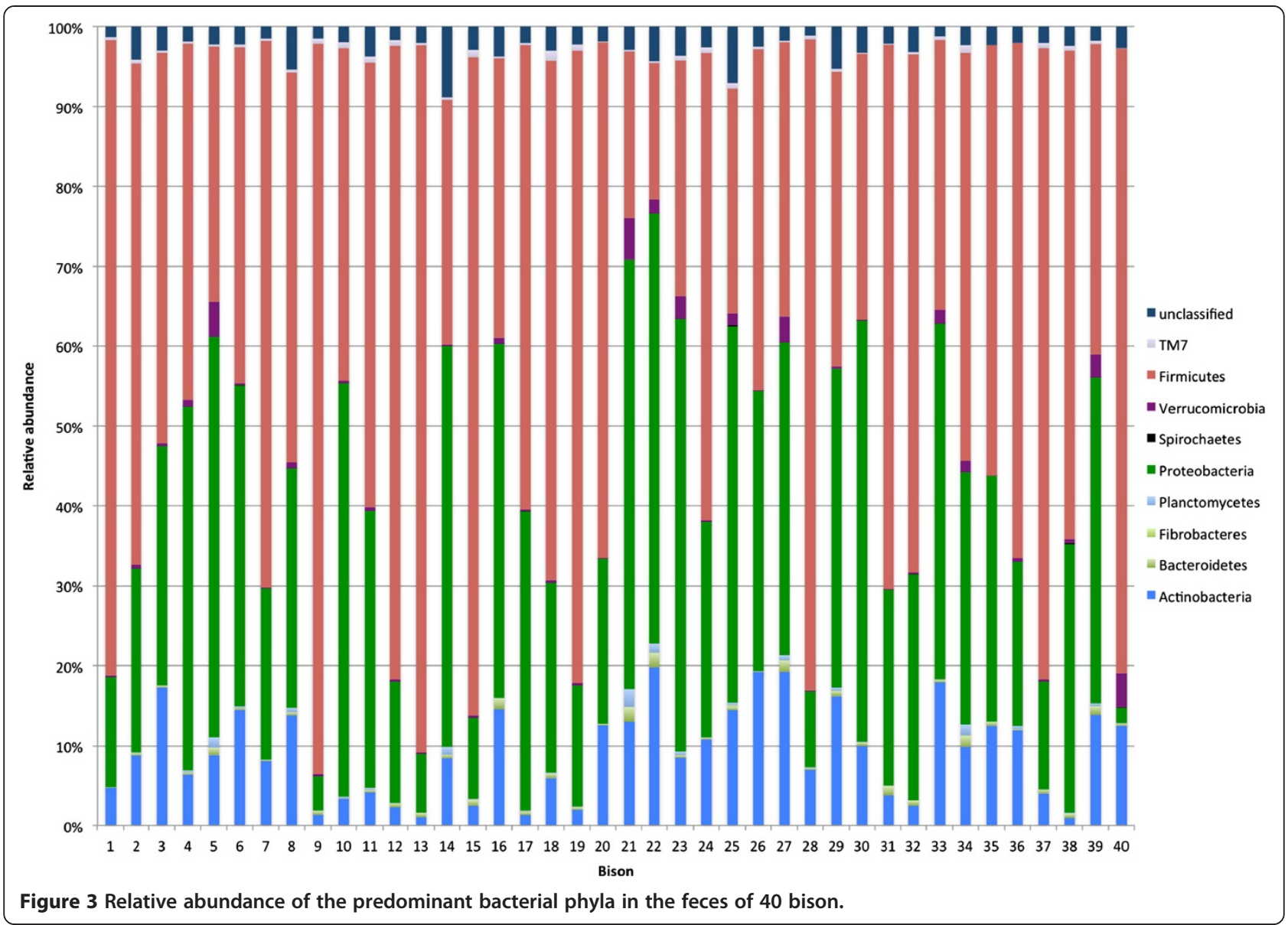

concept of 'enterotypes'. It has been proposed that the intestinal microbiota of most humans can be categorized into three enterotypes based on predominance of different bacterial groups, Bacteroides, Prevotella or Ruminococcus [11]. The basis behind this concept is that different population structures may be able to achieve similar function in the intestinal tract; however, it is unclear whether truly distinct enterotypes exist [12] and, if so, the relevance. Enterotypes have not been reported in other species, yet the presence of markedly different fecal microbiotas amongst similar animals in this study suggests this phenomenon might occur in bison. This could be further explored by study of the functional genetic composition of the microbiota rather than relying solely on phylogeny.

The clinical relevance of the group differences noted here is unclear. Consistent with the concept of enterotypes, it is possible that there is adequate functional redundancy so that even profound differences in microbial populations can achieve the same functional results in the intestinal tract. However, study of the potential health impacts of these different microbial populations is warranted. This is perhaps most relevant in Group B, considering the high prevalence of Proteobacteria and relatively low abundance of Firmicutes. Proteobacteria tend to be of much lower abundance in the feces of healthy ruminants and other species compared to the relative abundance noted here [13-16], and increases in Proteobacteria are often associated with 'dysbiosis' and intestinal inflammation [17-19]. Increases in the relative abundance of Proteobacteria have been reported with various types of intestinal tract disease in diverse species such as humans, horses and dogs $[13,14,18,20]$. This study cannot evaluate the health impacts of Proteobacteria or other groups in bison, but the striking differences and concern about this phylum in other species raise some questions that should be addressed further. In contrast, the high abundance of Firmicutes in Group A is consistent with previous reports in ruminants $[15,16,21]$ and hindgut fermenters $[14,22]$. This phylum consists of a broad range of Gram positive bacteria with diverse functions and clinical relevance. It includes a large number of species with important roles in digestion and energy conversion and is expected to be present in large numbers.

The dominance of a small number of phyla is consistent with other fecal microbiota studies, where a large number of phyla may be present with only a few accounting for more than $1 \%$ of sequences. Typically, Firmicutes, Bacteroidetes, Proteobacteria, Actinobacteria and Verrucomicrobia 


\begin{tabular}{|c|c|c|c|}
\hline Phylum & Group A $(n=19)$ & Group B $(n=21)$ & $P$ value \\
\hline Acidobacteria & 0.00008 & 0.00004 & 0.43 \\
\hline Actinobacteria & 6.4 & 1.2 & 0.002 \\
\hline Bacteroidetes & 0.49 & 0.65 & 0.26 \\
\hline Chlamydiae & 0.018 & 0.036 & 0.24 \\
\hline Chloroflexi & 0.0029 & 0.013 & 0.014 \\
\hline Deferibacteres & 0 & 0.00005 & 0.033 \\
\hline Deinococcus-Thermus & 0.00004 & 0.0023 & 0.40 \\
\hline Elusimicrobia & 0.000013 & 0.000008 & 0.72 \\
\hline Fibrobacteres & 0.016 & 0.018 & 0.80 \\
\hline Fusobacteria & 0.0029 & 0.0022 & 0.67 \\
\hline Gemmatimonadetes & 0.007 & 0.15 & 0.038 \\
\hline Lentispaerae & 0.0028 & 0.00005 & 0.24 \\
\hline Plantomycetes & 0.15 & 0.42 & 0.065 \\
\hline Proteobacteria & 18.7 & 42.5 & $<0.0001$ \\
\hline Spirochaetes & 0.035 & 0.022 & 0.35 \\
\hline Synergistetes & 0.0001 & 0.0018 & 0.24 \\
\hline Tenericutes & 0.0015 & 0.0030 & 0.15 \\
\hline Verrucomicrobia & 0.54 & 1.3 & 0.056 \\
\hline Firmicutes & 70.9 & 39.3 & $<0.001$ \\
\hline SR1 & 0 & 0.000055 & 0.11 \\
\hline TM7 & 0.42 & 0.42 & 0.97 \\
\hline Unclassified & 2.3 & 3.3 & 0.06 \\
\hline
\end{tabular}

Phyla that were significantly different area indicated in bold.

are most common [14,23-26], as was the case here. The relative abundance of Bacteroidetes was low compared to studies of cattle $[15,16,21]$; however, care must be taken when making such comparisons and comparison of bison and cattle samples using the same laboratory and analytical methods is required to evaluate potential differences between those species.

Three percent of genera accounted for $71 \%$ of sequences, yet no OTUs were found in all samples at a minimum relative abundance of $1 \%$. Significant differences between the two Groups for most of the main genera may be one explanation of the lack of an identified core microbiota between all bison. One Clostridium cluster XI OUT was the closest to a true core component, being found in all Group A samples and $90 \%$ of Group B samples as $1 \%$ of more of the sequences. Clostridium cluster XI has been reported to be a common constituent of the fecal microbiota of a diverse range of species, including dogs, cats, pigs, grizzly bears and humans [27-30], although it has received limited investigation. It has been suggested that Clostridium cluster XI levels may correlate with dietary protein in carnivores; [29], however, this group also includes many saccharolytic organisms [31], something that might account for the relatively high abundance in hay-fed bison. It is likely that this is a very broad group from a functional standpoint, with individual members that can play different roles in diverse gastrointestinal environments.

While this study can define microbial evenness and diversity, ideal values are not known so interpretation of these results in a clinical context is difficult. Greater diversity likely provides an added degree of functional redundancy, whereby there is a functional 'reserve' capacity in the microbiota to adapt to external influences. Yet, optimal diversity is unknown and some degree of unevenness (greater representation of some members of the microbiota) is expected, since some bacteria play more important roles than others (e.g. cellulolytic organisms should probably be present at greater abundances than many other species in a herbivore).

Comparison with previous culture-dependent study highlights the different results that can be obtained. One study of 96 bison samples involved a comprehensive culture-based approach yet only identified 19 'major' genera and a small but unclear number of lesser genera [4]. In contrast, 228 genera were identified in the current study, with species estimates exceeding 10000 per sample. Relative abundance was not evaluated in that study, but none of the ten most prevalence genera in the culturedependent study were amongst the most abundant genera identified here (Table 3). Rather than true differences in populations, these contrasts likely reflect the difficulty growing many of the common genera identified here by sequence-based methods and overgrowth-bias of potentially uncommon genera that are adept at growing under conventional culture conditions.

Fecal analyses must always be evaluated with an understanding that there are differences in the microbiota of different parts of the intestinal tract. This may be particularly important in bison, as the proximal location and markedly different environment of the rumen mean that feces do not closely reflect the state of the ruminal microbiota [16]. Yet, while these differences may limit the sensitivity of the use of feces for detection of alterations in proximal compartments, it is reasonable to assume that differences in the fecal microbiota indicate differences in at least some proximal locations, and that the intestinal (post-ruminal) microbiota is also important. Comparative study of the entire gastrointestinal tract can be informative [16] but requires either surgical collection of samples or collection immediately after euthanasia, both of which carry inherent logistical, cost and ethical issues. Concurrent study of the rumen and feces is more practical because rumen fluid collection is more feasible (although certainly not without challenges in bison). Future study comparing the rumen microbiota between different bison, and comparing the rumen and fecal 
Table 3 Relative abundance of predominant genera in the fecal microbiota of 40 bison

\begin{tabular}{|c|c|c|c|c|}
\hline Genus & Total & Group A & Group B & $P$ value \\
\hline Clostridium cluster XI & $13.1 \%$ & $17.5 \%$ & $8.3 \%$ & 0.004 \\
\hline Brevundimonas & $9.5 \%$ & $4.3 \%$ & $15.3 \%$ & $<0.001$ \\
\hline Unclassified Clostridiaceae & $8.9 \%$ & $10.1 \%$ & $7.4 \%$ & 0.013 \\
\hline Unclassified Lachnospiraceae & $6.6 \%$ & $7.8 \%$ & $5.1 \%$ & 0.023 \\
\hline Sedimentibacter & $4.2 \%$ & $5.9 \%$ & $1.9 \%$ & 0.001 \\
\hline Pseudomonas & $3.4 \%$ & $3.4 \%$ & $2.5 \%$ & 0.56 \\
\hline Unclassified Peptostreptococcaceae & $2.9 \%$ & $3.9 \%$ & $1.7 \%$ & 0.014 \\
\hline Unclassified bacterium & $2.8 \%$ & $2.4 \%$ & $3.3 \%$ & 0.055 \\
\hline Tissierella & $2.4 \%$ & $3.0 \%$ & $1.9 \%$ & 0.16 \\
\hline Devosia & $2.2 \%$ & $1.1 \%$ & $3.3 \%$ & 0.0007 \\
\hline Unclassified Clostridia & $1.7 \%$ & $2.0 \%$ & $1.5 \%$ & 0.065 \\
\hline Unclassified Comamonadaceae & $1.6 \%$ & $1.3 \%$ & $1.7 \%$ & 0.55 \\
\hline Nocardioides & $1.5 \%$ & $1.1 \%$ & $2.3 \%$ & 0.028 \\
\hline Phenylobacterium & $1.5 \%$ & $0.5 \%$ & $2.9 \%$ & 0.013 \\
\hline Pusillimonas & $1.5 \%$ & $1.0 \%$ & $1.9 \%$ & 0.006 \\
\hline Unclassified Ruminococcaceae & $1.4 \%$ & $1.9 \%$ & $0.8 \%$ & 0.002 \\
\hline Sporomusa & $1.4 \%$ & $2.5 \%$ & $0.7 \%$ & 0.008 \\
\hline Aeromicrobium & $1.3 \%$ & $0.9 \%$ & $1.8 \%$ & 0.18 \\
\hline Carnobacterium & $1.3 \%$ & $1.5 \%$ & $0.9 \%$ & 0.076 \\
\hline Unclassified Bacillales & $1.2 \%$ & $1.5 \%$ & $1.0 \%$ & 0.020 \\
\hline
\end{tabular}

microbiota would be useful. Further, this study also only involved one population of bison, so extrapolation to other populations of wild or captive bison, particularly those under different management and from different regions, must be done with care. Study of wild and captive bison populations from different regions would be useful to compare with the results obtained here and to help determine the 'normal' microbiota and its variations.

\section{Conclusion}

Two distinct microbial populations were identified in a group of semi-free-ranging bison, with no apparent explanation for the differences. Numerous bacterial phyla were identified, yet a small number accounted for the vast majority of the microbiota. Further study of the potential that distinct enterotypes may exist in the bison fecal microbiota, and if so, the clinical implications of such as phenomenon, is indicated to further explore the potential impact of this on bison health.

\section{End notes}

${ }^{a}$ E.Z.N.A. Stool DNA Kit, Omega Bio-Tek Inc., Doraville, GA, USA.

${ }^{\mathrm{b}}$ NanoDrop, Roche, Mississauga, Canada.

'Beckman Coulter Inc, Mississauga, Ontario, Canada.

dIllumina, San Diego, CA, USA.

\section{Availability of supporting data}

The dataset supporting the results of this article is available at the MG-RAST metagenomics analysis server (project TBA, http://metagenomics.anl.gov).

\section{Additional file}

Additional file 1: Figure S1. Rarefaction curves from assessment of the fecal microbiota of forty bison.

\section{Competing interest}

The authors declare that they have no competing interests.

\section{Authors' contribution}

JSW, TS and MJ designed the study. TS coordinated fecal collection. JSW directed laboratory testing, performed data analysis and prepared the manuscript. All authors read and approved the final manuscript.

\section{Acknowledgement}

This study was supported by a National Science and Engineering Research Council (NSERC) Discovery Grant and through the Canadian Institutes of Health Research (CIHR) Canada Research Chair program.

\section{Author details}

'Department of Pathobiology and Centre for Public Health and Zoonoses, Ontario Veterinary College, University of Guelph, Guelph, ON, Canada. ${ }^{2}$ Parks Canada, Saskatoon, SASK, Canada. ${ }^{3}$ Department of Veterinary Pathology, Western College of Veterinary Medicine, University of Saskatchewan, Saskatoon, SASK, Canada. ${ }^{4}$ Department of Large Animal Clinical Sciences, Western College of Veterinary Medicine, University of Saskatchewan, Saskatoon, SASK, Canada. 
Received: 19 March 2014 Accepted: 22 May 2014

Published: 28 May 2014

\section{References}

1. Towne G, Nagaraja TG, Cochran RC: Ruminal microbial populations and fermentation characteristics in bison and cattle fed high- and lowquality forage. Microb Ecol 1989, 17(3):311-316.

2. Towne G, Nagaraja TG, Cochran RC, Harmon DL, Owensby CE, Kaufman DW: Comparisons of ruminal fermentation characteristics and microbial populations in bison and cattle. Appl Environ Microbiol 1988, 54(10):2510-2514

3. Varel $\mathrm{VH}$, Dehority BA: Ruminal cellulolytic bacteria and protozoa from bison, cattle-bison hybrids, and cattle fed three alfalfa-corn diets. Appl Environ Microbiol 1989, 55(1):148-153.

4. Woodbury MR, Chirino-Trejo M: A survey of the fecal bacteria of bison (Bison bison) for potential pathogens and antimicrobial susceptibility of bison-origin E. coli. Can Vet J 2011, 52(4):414-418.

5. Klindworth A, Pruesse E, Schweer T, Peplies J, Quast C, Horn M, Glöckner FO: Evaluation of general $16 \mathrm{~S}$ ribosomal RNA gene PCR primers for classical and next-generation sequencing-based diversity studies. Nucleic Acids Res 2013, 41(1):e1

6. Eshar D, Weese JS: Molecular analysis of the microbiota in hard feces from healthy rabbits (Oryctolagus cuniculus) medicated with long term oral meloxicam. BMC Vet Res 2014, 10(1):62.

7. Schloss PD, Westcott SL, Ryabin T, Hall JR, Hartmann M, Hollister EB, Lesniewski RA, Oakley BB, Parks DH, Robinson CJ, Sahl JW, Stres B, Thallinger GG, Van Horn DJ, Weber CF: Introducing mothur: open-source, platform-independent, community-supported software for describing and comparing microbial communities. Appl Environ Microbiol 2009, 75(23):7537-7541.

8. Quast C, Pruesse E, Yilmaz P, Gerken J, Schweer T, Yarza P, Peplies J, Glöckner FO: The SILVA ribosomal RNA gene database project: improved data processing and web-based tools. Nucleic Acids Res 2013, 41:D590-D596.

9. Edgar RC, Haas BJ, Clemente JC, Quince C, Knight R: UCHIME improves sensitivity and speed of chimera detection. Bioinformatics 2011, 27(16):2194-2200

10. Bunge J: Estimating the number of species with CatchAll. In Pacific Symposium on Biocomputing Pacific Symposium on Biocomputing. 2011:121-130

11. Arumugam M, Raes J, Pelletier E, Le Paslier D, Yamada T, Mende DR, Fernandes GR, Tap J, Bruls T, Batto J-M, Bertalan M, Borruel N, Casellas F, Fernandez L, Gautier L, Hansen T, Hattori M, Hayashi T, Kleerebezem M, Kurokawa K, Leclerc M, Levenez F, Manichanh C, Nielsen HB, Nielsen T, Pons N, Poulain J, Qin J, Sicheritz-Ponten T, Tims S, et al: Enterotypes of the human gut microbiome. Nature 2011, 473(7346):174-180.

12. Huse SM, Ye Y, Zhou Y, Fodor AA: A core human microbiome as viewed through 16S rRNA sequence clusters. PLoS One 2012, 7(6):e34242.

13. Suchodolski JS, Xenoulis PG, Paddock CG, Steiner JM, Jergens AE: Molecular analysis of the bacterial microbiota in duodenal biopsies from dogs with idiopathic inflammatory bowel disease. Vet Microbiol 2010, 142(3-4):394-400.

14. Costa MC, Arroyo LG, Allen-Vercoe E, Stämpfli HR, Kim PT, Sturgeon A, Weese JS: Comparison of the Fecal Microbiota of healthy horses and horses with Colitis by high throughput sequencing of the V3-V5 region of the 16S rRNA gene. PLoS One 2012, 7(7):e41484.

15. Kim M, Kim J, Kuehn LA, Bono JL, Berry ED, Kalchayanand N, Freetly HC, Benson AK, Wells JE: Investigation of bacterial diversity in the feces of cattle fed different diets. J Anim Sci 2013. ePub ahead of print.

16. de Oliveira MNV, Jewell KA, Freitas FS, Benjamin LA, Tótola MR, Borges AC, Moraes $C A$, Suen $\mathrm{G}$ : Characterizing the microbiota across the gastrointestinal tract of a Brazilian Nelore steer. Vet Microbiol 2013, 164(3-4):307-314.

17. Kaakoush NO, Day AS, Huinao KD, Leach ST, Lemberg DA, Dowd SE, Mitchell HM: Microbial dysbiosis in pediatric patients with Crohn's disease. J Clin Microbiol 2012, 50(10):3258-3266.

18. Suchodolski JS, Dowd SE, Wilke V, Steiner JM, Jergens AE: 16S rRNA gene pyrosequencing reveals bacterial dysbiosis in the duodenum of dogs with idiopathic inflammatory bowel disease. PLoS One 2012, 7(6):e39333.

19. Winter SE, Bäumler AJ: Why related bacterial species bloom simultaneously in the gut: principles underlying the 'Like will to like'concept. Cell Microbiol 2013, 16(2):179-184.

20. Shahinas D, Silverman M, Sittler T, Chiu C, Kim P, Allen-Vercoe E, Weese S, Wong A, Low DE, Pillai DR: Toward an understanding of changes in diversity associated with fecal microbiome transplantation based on $16 \mathrm{~S}$ rRNA gene deep sequencing. MBio 2012, 23(3):e00338-00312.

21. Rudi K, Moen B, Sekelja M, Frisli T, Lee MRF: An eight-year investigation of bovine livestock fecal microbiota. Vet Microbiol 2012, 160(3-4):369-377.

22. O' Donnell MM, Harris HMB, Jeffery IB, Claesson MJ, Younge B, O' Toole PW, Ross RP: The core faecal bacterial microbiome of Irish Thoroughbred racehorses. Lett Appl Microbiol 2013, 57(6):492-501.

23. Steelman SM, Chowdhary BP, Dowd S, Suchodolski J, Janečka JE: Pyrosequencing of $16 \mathrm{~S}$ rRNA genes in fecal samples reveals high diversity of hindgut microflora in horses and potential links to chronic laminitis. BMC Vet Res 2012, 8:231.

24. Handl S, German A, Holden S, Dowd S, Steiner J, Heilmann R, Grant R, Swanson K, Suchodolski J: Fecal microbiota in lean and obese dogs. FEMS Microbiol Ecol 2013, 84(2):332-343.

25. Chaban B, Links MG, Hill JE: A molecular enrichment strategy based on cpn60 for detection of Epsilon-Proteobacteria in the dog fecal microbiome. Microb Ecol 2011, 63(2):348-357.

26. Swanson KS, Dowd SE, Suchodolski JS, Middelbos IS, Vester BM, Barry KA, Nelson KE, Torralba M, Henrissat B, Coutinho PM, Cann IK, White BA, Fahey GC: Phylogenetic and gene-centric metagenomics of the canine intestinal microbiome reveals similarities with humans and mice. ISME J 2010, 5(4):639-649.

27. Handl S, Dowd SE, Garcia-Mazcorro JF, Steiner JM, Suchodolski JS: Massive parallel $16 \mathrm{~S}$ rRNA gene pyrosequencing reveals highly diverse fecal bacterial and fungal communities in healthy dogs and cats. FEMS Microbiol Ecol 2011, 76(2):301-310.

28. Mori K, Ito T, Miyamoto H, Ozawa M, Wada S, Kumagai Y, Matsumoto J, Naito R, Nakamura S, Kodama H, Kurihara Y: Oral administration of multispecies microbial supplements to sows influences the composition of gut microbiota and fecal organic acids in their post-weaned piglets. J Biosci Bioeng 2011, 112(2):145-150.

29. Schwab C, Cristescu B, Northrup JM, Stenhouse GB, Gänzle M: Diet and environment shape fecal bacterial microbiota composition and enteric pathogen load of grizzly bears. PLoS One 2011, 6(12):e27905

30. Song Y, Liu C, Finegold SM: Real-time PCR quantitation of clostridia in feces of autistic children. Appl Environ Microbiol 2004, 70(11):6459-6465.

31. Collins MD, Lawson PA, Willems A, Cordoba JJ, Fernandez-Garayzabal J, Garcia P, Cai J, Hippe H, Farrow JA: The phylogeny of the genus Clostridium: proposal of five new genera and eleven new species combinations. Int J Syst Bacteriol 1994, 44(4):812-826.

doi:10.1186/1746-6148-10-120

Cite this article as: Weese et al:: The fecal microbiota of semi-free-ranging wood bison (Bison bison athabascae). BMC Veterinary Research 2014 10:120.

\section{Submit your next manuscript to BioMed Central and take full advantage of:}

- Convenient online submission

- Thorough peer review

- No space constraints or color figure charges

- Immediate publication on acceptance

- Inclusion in PubMed, CAS, Scopus and Google Scholar

- Research which is freely available for redistribution 